\title{
METRIC INHOMOGENEOUS DIOPHANTINE APPROXIMATION IN POSITIVE CHARACTERISTIC
}

\author{
S. KRISTENSEN*
}

\begin{abstract}
We obtain asymptotic formulae for the number of solutions to systems of inhomogeneous linear Diophantine inequalities over the field of formal Laurent series with coefficients from a finite fields, which are valid for almost every such system. Here 'almost every' is with respect to Haar measure of the coefficients of the homogeneous part when the number of variables is at least two (singly metric case), and with respect to the Haar measure of all coefficients for any number of variables (doubly metric case). As consequences, we derive zero-one laws in the spirit of the KhintchineGroshev Theorem and zero-infinity laws for Hausdorff measure in the spirit of Jarník's Theorem. The latter result depends on extending a recently developed slicing technique of Beresnevich and Velani to the present setup.
\end{abstract}

\section{Introduction and background}

The fields of formal Laurent series with coefficients from a finite field are a natural setting for studying questions about complexity and combinatorics, see e.g. [18]. It is also a natural setting for studying analogues of other areas of mathematics, with number theory being an important example of this. In the present paper, we study the metrical theory of Diophantine approximation over these fields.

This study originated with de Mathan [15], who among other things proved an analogue of Khintchine's celebrated theorem [8] from the real numbers. This was extended to systems of linear forms by the present author [9]. In that paper, the Hausdorff dimension of the involved exceptional sets is also calculated, providing an analogue of the Jarník-Besicovitch theorem in the setting of formal Laurent series.

A number of developments have occurred in metrical Diophantine approximation in this setting since the publication of [9]. At the same time, in the more familiar setting of the real numbers a number of new tools have been developed (e.g., [1], [2], [3]), and both classical and newer results now admit considerably simpler proofs.

\footnotetext{
* Research funded by the Carlsberg Foundation.

Received 26 June 2009.
} 
The purpose of the present paper is two-fold. One objective is to translate and extend where appropriate the recently developed methods mentioned above to the setting of formal Laurent series. The second objective is to derive analogues of several results of Schmidt [19], [20], in showing how a number of properties hold true for almost all systems of affine forms. We will also deduce Hausdorff dimension versions of some of these theorems. In particular, we extend results of Dickinson [5], Dodson [6] and Levesley [12] to the present setting. As a corollary of our results, we extend a recent result of $\mathrm{Ma}$ and $\mathrm{Su}$ [13] to higher dimensions.

In fact, our results represent a significant generalisation of that of Ma and $\mathrm{Su}$, as we obtain an asymptotic formula for the number of solutions to certain Diophantine inequalities including those studied in [13]. This extends results of Dodson, Levesley and the author [7] as well as Berthé, Nakada and Natsui [4] to the inhomogeneous setting. ${ }^{1}$

We now define the setup used throughout the paper. Let $\mathrm{F}$ denote the finite field of $q=p^{\ell}$ elements and consider the polynomial ring $\mathrm{F}[X]$ with coefficients from $\mathrm{F}$. We introduce an absolute value on $\mathrm{F}$ by letting $|P|=q^{\operatorname{deg}(P)}$ for $P \in \mathrm{F}[X] \backslash\{0\}$ and $|0|=0$. This induces an absolute value on the field of fractions $\mathrm{F}(X)$, which is in turn completed with respect to the absolute value to obtain the complete valued field $\mathrm{F}\left(\left(X^{-1}\right)\right)$. Evidently,

$$
\mathrm{F}\left(\left(X^{-1}\right)\right)=\left\{\sum_{i=-n}^{\infty} a_{-i} X^{-i}: a_{-i} \in \mathrm{F}, a_{n} \neq 0\right\} \cup\{0\},
$$

and in this representation,

$$
\left|\sum_{i=-n}^{\infty} a_{-i} X^{-i}\right|=q^{n}
$$

This representation of the elements in the field justifies the name 'formal Laurent series'.

In analogy with the integer part of the real numbers, we will denote by $[x]$ the polynomial part of $x \in \mathrm{F}\left(\left(X^{-1}\right)\right)$, i.e., the part of the expansion for which no negative exponents occur. We also define

$$
\mathscr{I}=\left\{x \in \mathrm{F}\left(\left(X^{-1}\right)\right):[x]=0\right\},
$$

\footnotetext{
${ }^{1}$ Note added in proof: A different extension of these results is to be found in M. Fuchs, Metrical theorems for inhomogeneous Diophantine approximations in positive characteristic, Acta Arith. 141 (2010), 191-208.
} 
the unit ball in $\mathrm{F}\left(\left(X^{-1}\right)\right)$. Additionally, we will let $\|x\|$ denote the distance from $x$ to the nearest polynomial in the metric induced by the absolute value defined above.

The field obtained in this way is ultra-metric, locally compact and has characteristic $p$, the characteristic of the base field $\mathrm{F}$. As a locally compact field, $\mathrm{F}\left(\left(X^{-1}\right)\right)$ supports a Haar measure, which is unique up to scaling by a positive constant. We let $\mu$ denote the Haar measure scaled in such a way that $\mu(\mathscr{I})=1$.

We will consider matrices and vector spaces over $\mathrm{F}\left(\left(X^{-1}\right)\right)$. More specifically, we will be working inside the 'unit cube', i.e., $\mathscr{I}^{h}$, where $h \in \mathrm{N}$. For these vector spaces, we will slightly abuse notation and let $|\mathbf{x}|=\max \left\{\left|x_{1}\right|, \ldots,\left|x_{h}\right|\right\}$ for $\mathbf{x}=\left(x_{1}, \ldots, x_{h}\right) \in \mathbf{F}\left(\left(X^{-1}\right)\right)^{h}$. Similarly, we will let $\|\mathbf{x}\|$ denote the distance to the nearest vector with polynomial coordinates in the metric induced by this norm. Throughout, we denote by $\operatorname{Mat}_{m, n}(\mathscr{I})$ the set of $m$ by $n$ matrices with entries from $\mathscr{I}$. We will identify $\operatorname{Mat}_{m, n}(\mathscr{I})$ with $\mathscr{I}^{m n}$, and equip the set with the $m n$-fold product measure of $\mu$, which we denote again by a slight abuse of notation by $\mu$.

Let $n, m \in \mathrm{N}$ be fixed. We will consider the Diophantine inequalities

$$
\|\mathbf{q} A-\mathbf{y}\|<\psi(|\mathbf{q}|),
$$

where $\mathbf{q} \in \mathrm{F}[X]^{m}, A \in \operatorname{Mat}_{m, n}(\mathscr{I})=\mathscr{I}^{m n}, \mathbf{y} \in \mathscr{I}^{n}$ and $\psi:\left\{q^{r}: r \in \mathrm{Z}\right\} \rightarrow$ $\left\{q^{r}: r \in \mathrm{Z}\right\}$ is some non-increasing function. We will be concerned with the question of the existence of infinitely many solutions to (1) as well as the asymptotic number of solutions the equation as $|\mathbf{q}|$ grows.

Most known results deal with the almost sure existence or non-existence of infinitely many solutions to (1). For our purposes, we define sets

$$
\begin{aligned}
& W_{m, n}(\psi, \mathbf{y})=\left\{A \in \operatorname{Mat}_{m, n}(\mathscr{I}):(1)\right. \text { has } \\
&\left.\infty \text { many solutions } \mathbf{q} \in \mathrm{F}[X]^{m}\right\},
\end{aligned}
$$

and

$$
\begin{aligned}
W_{m, n}(\psi)=\left\{(A, \mathbf{y}) \in \operatorname{Mat}_{m, n}(\mathscr{I}) \times\right. & \mathscr{I}^{n}:(1) \text { has } \\
& \left.\infty \text { many solutions } \mathbf{q} \in \mathrm{F}[X]^{m}\right\} .
\end{aligned}
$$

In (2), the vector $\mathbf{y}$ is fixed, and we refer to the study of the measure and dimension of this set as the singly metric theory. In (3), the inhomogeneous term $\mathbf{y}$ is varying, and we call this the doubly metric setup.

In this setup, much is known about $W_{m, n}(\psi, \mathbf{0})$, the homogeneous setup. The work of de Mathan [15] calculates the measure of $W_{1,1}(\psi, 0)$, while in [9] the Hausdorff dimension of this set as well as the measure of $W_{m, n}(\psi, \mathbf{0})$ is 
calculated for $m>1$. In [10], the Hausdorff dimension of the sets $W_{m, n}(\psi, \mathbf{0})$ is calculated without the assumption that $\psi$ is non-increasing. In [7], we calculate the asymptotics of the number of solutions to (1) when $\mathbf{y}=\mathbf{0}$ and $m>1$. The case $m=n=1$ was subsequently dealt with by Berthé, Nakada and Natsui [4].

It appears that the inhomogeneous theory, i.e., the case when $\mathbf{y} \neq \mathbf{0}$, has been somewhat neglected. An exception to this is the paper by $\mathrm{Ma}$ and $\mathrm{Su}$ [13], where the measure and Hausdorff dimension of $W_{1,1}(\psi)$ is calculated. It appears that the more difficult singly metric case has so far avoided study.

A main result in the present paper is the following asymptotic formula, which counts of the number of solutions to (1) for a fixed $\mathbf{y}$. The formula is valid for almost every $A \in \operatorname{Mat}_{m, n}(\mathscr{I})$.

Theorem 1.1. Let $m \geq 2$ and let $\mathbf{y} \in \mathrm{F}\left(\left(X^{-1}\right)\right)^{n}$ be fixed, let $\psi:\left\{q^{r}: r \in\right.$ $\mathrm{Z}\} \rightarrow\left\{q^{r}: r \in \mathrm{Z}\right\}$ be non-increasing. For $Q \in\left\{q^{t}: t \in \mathrm{N}\right\}$, let

$$
\Phi(Q)=\sum_{\substack{\mathbf{q} \in \mathrm{F}[X]^{m} \\ \mathbf{q} \leq Q}} \psi(\mathbf{q})^{n},
$$

and let $N(Q, A)$ denote the number of solutions to (1) with $|\mathbf{q}| \leq Q$. Then, for any $\epsilon>0$, for almost every $A \in \operatorname{Mat}_{m, n}(\mathscr{I})$ with respect to Haar measure,

$$
N(Q, A)=\Phi(Q)+O\left(\Phi(q)^{1 / 2} \log ^{3 / 2+\epsilon}(\Phi(Q))\right) .
$$

From this, we will derive asymptotics in the doubly metric case, where the result strengthens Corollary 1.4. Furthermore, we easily prove the result for the case $m=1$ as well and so obtain a complete result for the doubly metric case.

Corollary 1.2. Let $\psi:\left\{q^{r}: r \in \mathrm{Z}\right\} \rightarrow\left\{q^{r}: r \in \mathrm{Z}\right\}$ be non-increasing. For $Q \in\left\{q^{t}: t \in \mathrm{N}\right\}$, let

$$
\Phi(Q)=\sum_{\substack{\mathbf{q} \in F[X]^{m} \\ \mathbf{q} \leq Q}} \psi(\mathbf{q})^{n},
$$

and let $N(Q, A)$ denote the number of solutions to (1) with $|\mathbf{q}| \leq Q$. Then, for any $\epsilon>0$, for almost every $(A, \mathbf{y}) \in \operatorname{Mat}_{m, n}(\mathscr{I}) \times \mathscr{I}^{n}$ with respect to Haar measure,

$$
N(Q, A)=\Phi(Q)+O\left(\Phi(q)^{1 / 2} \log ^{3 / 2+\epsilon}(\Phi(Q))\right) .
$$


With these two results in place, we are able to deduce a number of results relating to the sets $W_{m, n}(\psi, \mathbf{y})$ and $W_{m, n}(\psi)$. We may instantly deduce the following result on the Haar measure of $W_{m, n}(\psi, \mathbf{y})$.

Theorem 1.3. Let $m \geq 2$ and let $\psi:\left\{q^{r}: r \in \mathrm{Z}_{\geq 0}\right\} \rightarrow\left\{q^{r}: r \in \mathrm{Z}\right\}$ be non-increasing. For any $\mathbf{y} \in \mathscr{I}^{n}$,

$$
\mu\left(W_{m, n}(\psi, \mathbf{y})\right)= \begin{cases}1 & \text { if } \sum_{\mathbf{q} \in \mathrm{F}[X]^{m}} \psi(|\mathbf{q}|)^{n}=\infty, \\ 0 & \text { if } \sum_{\mathbf{q} \in \mathrm{F}[X]^{m}} \psi(|\mathbf{q}|)^{n}<\infty .\end{cases}
$$

A generalisation of the Theorem 1.4 in $\mathrm{Ma}$ and $\mathrm{Su}$ [13] is an immediate consequence of Theorem 1.3 together with Fubini's Theorem. This result is proved without the restriction on $m$, i.e., for any $m \in \mathbf{N}$.

COROLlaRY 1.4. Let $\psi:\left\{q^{r}: r \in \mathrm{Z}_{\geq 0}\right\} \rightarrow\left\{q^{r}: r \in \mathrm{Z}\right\}$ be non-increasing. Then,

$$
\mu\left(W_{m, n}(\psi)\right)= \begin{cases}1 & \text { if } \sum_{\mathbf{q} \in \mathrm{F}[X]^{m}} \psi(|\mathbf{q}|)^{n}=\infty \\ 0 & \text { if } \sum_{\mathbf{q} \in \mathrm{F}[X]^{m}} \psi(|\mathbf{q}|)^{n}<\infty\end{cases}
$$

In fulfilling the first objective of the paper, we introduce a number of tools in the next section. Whenever the required result is already known, we quote it without proof. However, in one particular instance, we will need to extend the so-called Slicing Theorem of Beresnevich and Velani [3] to the present setup. With this result in place, we are in addition to the results for Haar measure able to give complete results for Hausdorff measures. For readers not aquainted with Hausdorff measures, the definition is given below in \$2.1 along with the definition of Hausdorff dimension. For a dimension function $f$, we will denote the Hausdorff $f$-measure by $\mathscr{H}^{f}$.

Theorem 1.5. Let $m \geq 2$ and let $\psi:\left\{q^{r}: r \in \mathbf{Z}_{\geq 0}\right\} \rightarrow\left\{q^{r}: r \in \mathbf{Z}\right\}$ be non-increasing and let $f$ be a dimension function such that $f(r) / r^{m n}$ is monotonic, and such that $g(r)=r^{-(m-1) n} f(r)$ is a dimension function. Then for any $\mathbf{y} \in \mathscr{I}^{n}$,

$$
\mu\left(W_{m, n}(\psi, \mathbf{y})\right)= \begin{cases}\mathscr{H}^{f}\left(\mathscr{I}^{m n}\right) & \text { if } \sum_{\mathbf{q} \in \mathrm{F}[X]^{m} \backslash\{0\}} g\left(\frac{\psi(|\mathbf{q}|)}{|\mathbf{q}|}\right)|\mathbf{q}|^{n}=\infty, \\ 0 & \text { if } \sum_{\mathbf{q} \in \mathrm{F}[X]^{m} \backslash\{0\}} g\left(\frac{\psi(|\mathbf{q}|)}{|\mathbf{q}|}\right)|\mathbf{q}|^{n}<\infty .\end{cases}
$$

Using the exact same derivation, which leads us to deduce Theorem 1.5 from Theorem 1.3, we may deduce a Hausdorff measure version of Corollary 1.4. 
COROLlary 1.6. Let $\psi:\left\{q^{r}: r \in \mathrm{Z}_{\geq 0}\right\} \rightarrow\left\{q^{r}: r \in \mathrm{Z}\right\}$ be non-increasing and let $f$ be a dimension function such that $f(r) / r^{(m+1) n}$ is monotonic, and such that $g(r)=r^{-m n} f(r)$ is a dimension function. Then,

$$
\mu\left(W_{m, n}(\psi)\right)= \begin{cases}\mathscr{H}^{f}\left(\mathscr{I}^{m n}\right) & \text { if } \sum_{\mathbf{q} \in \mathrm{F}[X]^{m} \backslash\{0\}} g\left(\frac{\psi(|\mathbf{q}|)}{|\mathbf{q}|}\right)|\mathbf{q}|^{n}=\infty, \\ 0 & \text { if } \sum_{\mathbf{q} \in \mathrm{F}[X]^{m} \backslash\{0\}} g\left(\frac{\psi(|\mathbf{q}|)}{|\mathbf{q}|}\right)|\mathbf{q}|^{n}<\infty\end{cases}
$$

Note that while the corollary looks almost identical to Theorem 1.5, there is a difference in the definition of the function $g$ occurring in the series. This is critical, and encodes the fact that there are more dimensions available in the corollary.

As a corollary of Theorem 1.5, we deduce an analogue of Levesley's result [12] in positive characteristic. To state it precisely, we need the definition of the order at infinity $\lambda(h)$ of a function $h$. Let $h: \mathbf{R}_{+} \rightarrow \mathbf{R}_{+}$be some function, and define

$$
\lambda(h)=\lim _{x \rightarrow \infty} \frac{\log h(x)}{\log x}
$$

whenever this limit exists. We will apply this definition to functions $h:\left\{q^{r}\right.$ : $r \in \mathrm{Z}\} \rightarrow\left\{q^{r}: r \in \mathrm{Z}\right\}$. In this case, we just take the limit over the set $\left\{q^{r}: r \in \mathrm{Z}\right\}$ as $r \rightarrow \infty$.

Corollary 1.7. Let $m \geq 2$ and let $\psi:\left\{q^{r}: r \in \mathrm{Z}\right\} \rightarrow\left\{q^{r}: r \in \mathrm{Z}\right\}$ be such that the order at infinity of the function $1 / \psi$ exists. Let $\lambda$ denote this quantity. Then for any $\mathbf{y} \in \mathscr{I}^{n}$,

$$
\operatorname{dim}_{H}\left(W_{m, n}(\psi, \mathbf{y})\right)= \begin{cases}(m-1) n+\frac{m+n}{1+\lambda} & \text { if } \lambda>\frac{m}{n} \\ m n & \text { if } \lambda \leq \frac{m}{n}\end{cases}
$$

Note that in Levesley's paper [12], the order at infinity of $1 / \psi$ is not required to exist. In that paper, the order at infinity is replaced with the lower order at infinity, which is in turn defined by replacing the limit with a lim inf in (4). Also, the requirement that $m \geq 2$ is not needed in Levesley's proof. No doubt the present result can be extended to this situation, but for the sake of clarity of exposition, we have chosen not to do this.

As a final corollary, we obtain a multidimensional generalisation of Theorem 1.5 in [13]. This result also extends results of Dickinson [5] and Dodson [6] to the setting of formal Laurent series. 
Corollary 1.8. Let $\psi:\left\{q^{r}: r \in \mathrm{Z}\right\} \rightarrow\left\{q^{r}: r \in \mathrm{Z}\right\}$ be such that the order at infinity of the function $1 / \psi$ exists. Let $\lambda$ denote this quantity. Then,

$$
\operatorname{dim}_{H}\left(W_{m, n}(\psi)\right)= \begin{cases}m n+\frac{m+n}{1+\lambda} & \text { if } \lambda>\frac{m}{n}, \\ (m+1) n & \text { if } \lambda \leq \frac{m}{n}\end{cases}
$$

The paper is structured as follows. In Section 2, we introduce the necessary tools for the proofs on the main theorems. We will require some standard results and definitions from measure theory and probability theory, which will be recalled in Section 2.1. We state a number of results from literature. Whenever a proof is not available in literature, we present such a proof here. Notably, in Section 2.4 we extend the recently developed slicing technique to the present setting.

In Section 3, we discuss asymptotic formulae, and prove Theorem 1.1 and Corollary 1.2. Together with the auxiliary results from Section 2, this will in turn allow us to deduce Theorem 1.3 and all the following consequences, i.e., Corollary 1.4, Theorem 1.5 and Corollaries 1.6-1.8. This is done in Section 4. Finally, in Section 5 we give some concluding remarks.

We will sometimes use the Vinogradov notation: For two real quantities $f$ and $g$, we will write $f \ll g$ if there exists a constant $c>0$ such that $f \leq c g$. An equivalent way of saying the same thing in Landau's $O$-notation is $f=O(g)$. If $f \ll g$ and $g \ll f$, we will write $f \asymp g$.

\section{Tools}

\subsection{Measure theory}

There will be two kinds of measures at work in this paper. One is the Haar measure on $\mathrm{F}\left(\left(X^{-1}\right)\right)$ and vector spaces over this field. The other is Hausdorff measures, which can be defined in arbitrary metric spaces, but will only be considered on $\mathrm{F}\left(\left(X^{-1}\right)\right)$ in this paper.

Haar measure on the field of formal Laurent series has been completely described. Sprindžuk [21] constructed this measure directly, and found that the measure of a ball of radius $q^{r}$ is equal to $q^{r+1}$. This scaling ensures that the measure of the unit ball $\mathscr{I}$ is equal to 1 . Mahler [14] also used volumes in the setting of formal series. While Mahler's construction differs from that of Sprindžuk, uniqueness of the Haar measure (up to a scaling constant) ensures that results of both papers are valid for the Haar measure.

We now briefly define Hausdorff measures. We will say that a function $f: \mathbf{R}_{\geq 0} \rightarrow \mathbf{R}_{\geq 0}$ is a dimension function if it is continuous and non-decreasing with $f(0)=0$. Given a dimension function $f$, the Hausdorff $f$-measure on a locally compact metric space $(X, d)$ is defined as follows. For a set $F \subseteq X$, 
consider the family $\mathscr{C}_{\rho}$ of countable open coverings $\mathscr{C}$ of $F$ by balls $B_{i}$ of radius $r\left(B_{i}\right) \leq \rho$, where $\rho>0$. The Hausdorff $f$-measure of $f$ is given by

$$
\mathscr{H}^{f}(F)=\lim _{\rho \rightarrow 0} \inf _{\mathscr{C} \in \mathscr{C}_{\rho}} \sum_{B_{i} \in \mathscr{C}} f\left(r\left(B_{i}\right)\right)=\sup _{\rho>0} \inf _{\mathscr{C} \in \mathscr{C}_{\rho}} \sum_{B_{i} \in \mathscr{C}} f\left(r\left(B_{i}\right)\right) .
$$

The final equality follows as the infimum can only increase when we impose additional restrictions on the covers over which it is taken.

If $f(r)=r^{s}$ for some $s>0$, we will denote the Hausdorff $f$-measure by $\mathscr{H}^{s}$ and speak of the Hausdorff $s$-measure. For a set $F$, the Hausdorff dimension of $F$ is defined by

$$
\operatorname{dim}_{H}(F)=\inf \left\{s>0: \mathscr{H}^{s}(F)=0\right\} .
$$

When the metric space in question is $\mathrm{R}^{h}$, the Hausdorff $h$-measure is comparable with the usual $h$-dimensional Lebesgue measure, so that sets of positive measure have Hausdorff dimension $h$. Similar statements hold for vector spaces over $\mathrm{F}\left(\left(X^{-1}\right)\right)$, where the measure is product of the Haar measure on the base field. Note that the Haar measure on each of these fields shares an important property with the Lebesgue measure on R: The Haar measure of a ball of radius $r$ is comparable with the radius of the ball.

Finally, we need a 'shrinking lemma', essentially stating that if the lim sup set of a sequence of balls is full, this property is preserved when we scale the balls by an absolute factor. Recall that if $\left\{B_{i}\right\} \subseteq X$ is a sequence of subsets of the set $X$, then

$$
\lim \sup B_{i}=\bigcap_{N=1}^{\infty} \bigcup_{i \geq N} B_{i}=\left\{x \in X: x \in B_{i} \text { for infinitely many } i \in \mathrm{N}\right\} .
$$

LEMma 2.1. Let $\left\{B_{i}\right\}$ be a sequence of balls in $\mathrm{F}\left(\left(X^{-1}\right)\right)^{h}$, let $U \subseteq \mathrm{F}\left(\left(X^{-1}\right)\right)^{h}$ be open and suppose that $\lim \sup B_{i}$ is full in $U$. Let $\delta \in(0,1)$ and denote by $\delta B_{i}$ the ball with the same centre as $B_{i}$ and with radius $\delta$ times the radius of $B_{i}$. Then $\lim \sup \delta B_{i}$ is full in $U$.

The proof of Lemma is identical to that of [3, Lemma 4.3], where it is deduced from [2, Lemma 6]. The only property of $\mathrm{R}^{h}$ used in that proof is that $\mathscr{H}^{h}$ is comparable to the $h$-dimensional Lebesgue measure. This holds true in the present setting on reading 'Haar' in place of 'Lebesgue'.

\subsection{Resonant neighbourhoods}

Two critical estimates in all proofs to follow concerns the measure of the socalled resonant neighbourhoods as well as the intersections of these. These are the sets of matrices where one particular inequality of the form (1) is satisfied. 
The term 'resonant' comes from physics, where resonance occurs when certain Diophantine equations have solutions. Here, we are merely close to a solution, which justifies the term 'neighbourhood'.

Let $m, n$ be fixed and fix an inhomogeneous term $\mathbf{y} \in \mathrm{F}\left(\left(X^{-1}\right)\right)^{n}$ and a $\mathbf{q} \in \mathrm{F}[X]^{m}$. We define sets

$$
\tilde{B}(\mathbf{q}, \epsilon)=\left\{A \in \operatorname{Mat}_{m, n}(\mathscr{I}):\|\mathbf{q} A-\mathbf{y}\|<\epsilon\right\},
$$

for any $\epsilon>0$. In our notation, we have suppressed the dependence on $\mathbf{y}$, which will be fixed in any concrete case. We will additionally suppose that $\epsilon \in\left\{q^{r}: r \in \mathrm{Z}\right\}$ in order to avoid ambiguities. We will reserve the notation $B(\mathbf{x}, \rho)$ for a ball centred at $\mathbf{x}$ of radius $\rho$.

We have the following estimates, from which all subsequent results will follow.

Lemma 2.2. With $\tilde{B}(\mathbf{q}, \epsilon)$ as in (5),

$$
\mu(\tilde{B}(\mathbf{q}, \epsilon))=\epsilon^{n} .
$$

Proof. We proceed as in [9]. The set $\tilde{B}(\mathbf{q}, \epsilon)$ is the disjoint union of neighbourhoods around the solutions to $\mathbf{q} A-\mathbf{y}=\mathbf{p}$, where $\mathbf{p} \in \mathrm{F}[X]^{n}$. Since we further require that $A \in \operatorname{Mat}_{m, n}(\mathscr{I})$, we immediately find that exactly $|\mathbf{q}|^{n}$ values of $\mathbf{p}$ give rise to solutions. Note that this uses the ultrametricity of the setup.

Now let $\mathbf{p}$ be fixed. We now have,

$$
\begin{aligned}
\mu\left\{A \in \operatorname{Mat}_{m, n}(\mathscr{I}):\|\mathbf{q} A-\mathbf{y}-\mathbf{p}\|<\epsilon\right\} & \\
& =\mu\left\{A \in \operatorname{Mat}_{m, n}(\mathscr{I}): \mathbf{q} A \in B(\mathbf{y}+\mathbf{p}, \epsilon)\right\}=\frac{\epsilon^{n}}{|\mathbf{q}|^{n}} .
\end{aligned}
$$

The latter equality follows by noting that the set in question is convex and hence a parallelepiped by [14]. The result now follows using Mahler's estimates [14]. Note again that ultrametricity is critical for the argument. The lemma now follows on taking the union of the above disjoint sets.

Lemma 2.3. Suppose that $m \geq 2$ and that $\mathbf{q}, \mathbf{q}^{\prime} \in \mathrm{F}[X]^{m}$ are linearly independent over $\mathrm{F}\left(\left(X^{-1}\right)\right)$. Then,

$$
\mu\left(\tilde{B}(\mathbf{q}, \epsilon) \cap \tilde{B}\left(\mathbf{q}^{\prime}, \epsilon^{\prime}\right)\right)=\mu(\tilde{B}(\mathbf{q}, \epsilon)) \mu\left(\tilde{B}\left(\mathbf{q}^{\prime}, \epsilon^{\prime}\right)\right) .
$$

Proof. Consider first the case $m=2, n=1$. In this case, the set $\tilde{B}(\mathbf{q}, \epsilon) \cap$ $\tilde{B}\left(\mathbf{q}^{\prime}, \epsilon^{\prime}\right)$ consists of disjoint parallelograms containing the intersection points of the lines $\mathbf{q} A-y-p_{1}=0$ and $\mathbf{q}^{\prime} A-y-p_{2}=0$. Mahler [14] calculated 
the measure of such sets. Indeed, he showed that the measure of each of the parallelograms must be $\epsilon \epsilon^{\prime} /\left|\operatorname{det}\left(\mathbf{q}, \mathbf{q}^{\prime}\right)\right|$, i.e., the product of the right hand sides of the defining inequalities divided by the determinant of the matrix with $\mathbf{q}$ and $\mathbf{q}^{\prime}$ as columns. Counting the number of components as in the proof of Lemma 2.2, we obtain the result in this special case.

For the general case, consider $n$ copies of the span of $\mathbf{q}$ and $\mathbf{q}^{\prime}$. In this way, we arrive at $\mathrm{nm}$-dimensional parallelepipeds, for which the measure may be calculated once more by Mahler's results. Counting the number of intersections yield the complete result.

When $m=1$, the situation is somewhat different. In that case, it is evidently not possible to have linear independence of two elements $q, q^{\prime} \in \mathrm{F}[X]$. Instead, one needs to consider a more arithmetic setup, and take into account the common divisors of $q$ and $q^{\prime}$. This in turn results in a weaker independence statement than that of Lemma 2.3.

In this paper, we will consider only the doubly metric setup for this case. This has the pleasing feature that we still have pairwise independence of the corresponding sets. We define the doubly metric resonant neighbourhoods

$$
\hat{B}(q, \epsilon)=\left\{(A, \mathbf{y}) \in \operatorname{Mat}_{1, n}(\mathscr{I}) \times \mathscr{I}^{n}:\|q A-\mathbf{y}\|<\epsilon\right\} .
$$

We may again calculate the measure of each set and their intersections.

Lemma 2.4. Suppose that $q, q^{\prime} \in \mathrm{F}[X]$ with $q \neq q^{\prime}$. Then,

$$
\mu(\hat{B}(q, \psi(|q|)))=\psi(|q|)^{n},
$$

and

$$
\mu\left(\hat{B}(q, \psi(|q|)) \cap \hat{B}\left(q^{\prime}, \psi(|q|)^{\prime}\right)\right)=\mu(\hat{B}(q, \psi(|q|))) \mu\left(\hat{B}\left(q^{\prime}, \psi(|q|)^{\prime}\right)\right) .
$$

Proof. This follows instantly from [13, Lemma 2.4] and Fubini's Theorem.

\subsection{The Mass Transference Principle}

One of the main techniques applied in this paper is the transfer of statements about the Haar measure of a lim sup set to statements about Hausdorff measures of the same set. In two papers [2], [3], Beresnevich and Velani developed tools for this purpose. In [2], a technique known as known as the Mass Transference Principle was developed for a large class of metric spaces. We quote the result here.

Theorem 2.5 ([2], Theorem 3 (The Mass Transference Principle)). Let $g: \mathbf{R}_{\geq 0} \rightarrow \mathbf{R}_{\geq 0}$ be a dimension function for which there is a $\lambda>1$ such 
that $f(2 x)>\lambda f(x)$ for all $x \in \mathbf{R}_{+}$. Let $(X, d)$ be a locally compact metric space such that for some $r_{0}>0$, for any ball $B \subseteq X$ of radius $r(B) \leq r_{0}$, $\mathscr{H}^{f}(B) \asymp g(r)$. For a ball $B=B(c, r) \subseteq X$ of centre $c$ and radius $r$ and $a$ dimension function $g$, we denote by $B^{g}$ the ball $B\left(c, f^{-1}(g(r))\right)$.

Let $\left\{B_{i}\right\}$ be a sequence of balls in $X$, such that $r\left(B_{i}\right) \rightarrow 0$ as $i \rightarrow \infty$, and let $g: \mathbf{R}_{\geq 0} \rightarrow \mathbf{R}_{\geq 0}$ be a dimension function such that $g(x) / f(x)$ is monotonic. Suppose that for any ball $B \subseteq X$,

$$
\mathscr{H}^{g}\left(B \cap \limsup _{i \rightarrow \infty} B_{i}^{f}\right)=\mathscr{H}^{g}(B) .
$$

Then, for any ball $B \subseteq X$,

$$
\mathscr{H}^{f}\left(B \cap \limsup _{i \rightarrow \infty} B_{i}^{g}\right)=\mathscr{H}^{f}(B) .
$$

\subsection{Slicing for local fields}

The purpose of this section is to extend the results of Beresnevich and Velani [3] to the present setup. In the original paper, the argument was only given for lim sup sets in vector spaces over the reals. We extend this to vector spaces over $\mathrm{F}\left(\left(X^{-1}\right)\right)$. The argument carries over to the $p$-adic fields without problems, but we will not need that here.

Let $h \in \mathrm{N}$ and let $\left\{R_{\alpha}\right\}$ be a countable collection of subsets of $\mathrm{F}\left(\left(X^{-1}\right)\right)^{h}$. To each $R_{\alpha}$, suppose that we have an associated weight $\beta_{\alpha} \in\left\{q^{r}: r \in \mathrm{Z}\right\}$ and let $\phi:\left\{q^{r}: r \in \mathrm{Z}\right\} \rightarrow\left\{q^{r}: r \in \mathrm{Z}\right\}$ be some function. We are interested in the set

$W\left(\left\{R_{\alpha}\right\}, \phi\right)=\left\{x \in \mathrm{F}\left(\left(X^{-1}\right)\right)^{h}: d\left(x, R_{\alpha}\right)<\phi\left(\beta_{\alpha}\right)\right.$ for infinitely many $\left.R_{\alpha}\right\}$.

Clearly, the sets studied in this paper are of this form.

In the following, let $h, t \in \mathrm{N}$ and $l \in \mathrm{Z}_{+}$be fixed with $h=l+t$. We let $\mathscr{R}=\left\{R_{\alpha}\right\}_{\alpha \in J}$ be a countable family of affine $l$-dimensional subspaces of an $h$-dimensional vector space over $\mathrm{F}\left(\left(X^{-1}\right)\right)$, i.e., sets of the form $V+b$ where $V \subseteq \mathrm{F}\left(\left(X^{-1}\right)\right)^{h}$ is an $l$-dimensional linear subspace and $b \in \mathrm{F}\left(\left(X^{-1}\right)\right)^{h}$. To each $R_{\alpha}$ and each $\delta>0$, we associate the $\delta$-neighbourhood of $R_{\alpha}$,

$$
\Delta\left(R_{\alpha}, \delta\right)=\left\{x \in \mathrm{F}\left(\left(X^{-1}\right)\right)^{h}: d\left(x, R_{\alpha}\right)<\delta\right\} .
$$

Associate to each $\alpha \in J$ a weight $\beta_{\alpha}>0$ and let $\phi:\left\{q^{r}: r \in \mathrm{Z}\right\} \rightarrow\left\{q^{r}\right.$ : $r \in \mathrm{Z}\}$ be a function. Suppose for every $\epsilon>0$ that $\#\left\{\alpha \in J: \beta_{\alpha}>\epsilon\right\}<\infty$. Finally, let $W\left(\left\{R_{\alpha}\right\}, \phi\right)$ be defined as in (7).

The following is our main result in this section. If we replace $\mathrm{F}\left(\left(X^{-1}\right)\right)$ with $\mathrm{R}$, this is [3, Theorem 3]. 
Theorem 2.6 (The Slicing Theorem). Let $h, l, t, \mathscr{R}$ and $\phi$ be as above. Let $V \subseteq \mathrm{F}\left(\left(X^{-1}\right)\right)^{h}$ be an $t$-dimensional linear subspace with

(i) $V \cap R_{\alpha} \neq \emptyset$ for any $\alpha \in J$,

(ii) $\sup _{\alpha \in J} \operatorname{diam}\left(V \cap \Delta\left(R_{\alpha}, 1\right)\right)<\infty$.

Let $f, g: \mathbf{R}_{\geq 0} \rightarrow \mathbf{R}_{\geq 0}$ be dimension functions with $g(r)=r^{-l} f(r)$, such that $r^{-h} f(r)$ is monotonic. Let $\Omega$ be a ball in $\mathrm{F}\left(\left(X^{-1}\right)\right)^{h}$ and suppose that for any ball $B \subseteq \Omega$,

$$
\mathscr{H}^{h}\left(B \cap W\left(\left\{R_{\alpha}\right\},(g \circ \phi)^{1 / t}\right)\right)=\mathscr{H}^{h}(B) .
$$

Then

$$
\mathscr{H}^{f}\left(B \cap W\left(\left\{R_{\alpha}\right\}, \phi\right)\right)=\mathscr{H}^{f}(B) .
$$

In order to prove Theorem 2.6, we will need the previously established auxiliary results as well as a lemma from fractal geometry.

In the following, we will use the notation $V^{\perp}$ and speak of 'orthogonal complements'. This is strictly speaking not the correct terminology, since the spaces considered do not have an inner product. What we mean by $V^{\perp}$ of a subspace $V$ is the following. Take a basis for a $k-l$-dimensional subspace $V \subseteq \mathrm{F}\left(\left(X^{-1}\right)\right)^{h}, v_{1}, \ldots, v_{k-l}$ and extend it to a basis for $V$ by adding to it vectors $v_{k-l+1}, \ldots, v_{h}$. We will let $V^{\perp}=\operatorname{span}\left\{v_{k-l+1}, \ldots, v_{h}\right\}$. Note that this is not a canonical choice, and the subspace $V^{\perp}$ depends on the procedure. This however causes no problems in the proofs.

In addition to the Mass Transference Principle, we will need an extension of part of [16, Theorem 10.10] to the setting of locally compact fields.

Lemma 2.7. Let $l, h \in \mathrm{N}$ with $l \leq h$, let $f, g: \mathbf{R}_{\geq 0} \rightarrow \mathbf{R}_{\geq 0}$ be dimension functions with $g(r)=r^{-l} f(r)$ and let $A \subseteq \mathrm{F}\left(\left(X^{-1}\right)\right)^{h}$ be a Borel set with $\mathscr{H}^{f}(A)<\infty$. Then for any $(k-l)$-dimensional linear subspace $V \subseteq \mathrm{F}\left(\left(X^{-1}\right)\right)^{h}$,

$$
\mathscr{H}^{g}(A \cap(V+a))<\infty
$$

for any $a \in V^{\perp} \backslash E$, where $\mathscr{H}^{l}(E)=0$.

PROof. The proof is identical to that in [16] or [3]. We need only observe that Fatou's Lemma holds for locally compact spaces (see e.g. [17, § I.9]). This implies that proof of Lemma 2.3 in [3] may now be copied (almost) verbatim. Lemma 2.7 now follows as an easy corollary.

We will use the contrapositive form of Lemma 2.7, which is the appropriate analogue of the Slicing Lemma of [3].

Lemma 2.8. Let $l, h \in \mathbf{N}$ with $l \leq h$, let $f, g: \mathbf{R}_{\geq 0} \rightarrow \mathbf{R}_{\geq 0}$ be dimension functions with $g(r)=r^{-l} f(r)$ and let $A \subseteq \mathrm{F}\left(\left(X^{-1}\right)\right)^{h}$ be a Borel set. Suppose 
that there is a set $S \subseteq V^{\perp}$ with $\mathscr{H}^{l}(S)>0$, such that for any $a \in S$,

$$
\mathscr{H}^{g}(A \cap(V+a))=\infty .
$$

Then, $\mathscr{H}^{f}(A)=\infty$.

With these tools in place, we can prove Theorem 2.6.

The proof falls in two cases. The first is the case when $l=0$, so that the $\mathscr{R}_{\alpha}$ are points. In this case, we easily deduce the result from Theorem 2.5. We subsequently deduce the full theorem, using again Theorem 2.5 and Lemma 2.8.

We make a first reduction of the number of cases to be considered. Let $B \subseteq \mathrm{F}\left(\left(X^{-1}\right)\right)^{h}$ be a fixed ball It is simple to observe, that if $r^{-h} f(r) \rightarrow 0$ as $r \rightarrow 0$, then $\mathscr{H}^{f}(B)=0$, and the result is immediate. If on the other hand $r^{-h} r(t) \rightarrow L$ as $r \rightarrow 0$ with $L$ positive and finite, the measures $\mathscr{H}^{f}$ and $\mathscr{H}^{h}$ are comparable, and the statement of the theorem is trivial. Hence, we assume throughout that

$$
r^{-h} f(r) \rightarrow \infty \quad \text { as } \quad r \rightarrow 0 .
$$

2.4.1. The case $l=0$. As $h=t, V=\mathrm{F}\left(\left(X^{-1}\right)\right)^{h}$, so clearly (i) holds. Now, for any $R_{\alpha}, \operatorname{diam}\left(V \cap \Delta\left(R_{\alpha}, 1\right)\right) \leq 2<\infty$, so (ii) is also trivially satisfied. Now, note that $(g \circ \phi)^{1 / t}(r)=f(\phi(r))^{1 / t}$, so that $W\left(\left\{R_{\alpha}\right\},(g \circ \phi)^{1 / t}\right)=$ $\lim \sup B_{i}^{f}$, where the $B_{i}$ are balls of radius $\phi(r)$. The result may now be read off directly from Theorem 2.5 on letting $g(r)=r^{h}$.

2.4.2. The case $l>0$. Under assumption $(8), \mathscr{H}^{h}(B)=\infty$, so the assumption of the theorem reads

$$
\mathscr{H}^{h}\left(B \cap W\left(\left\{R_{\alpha}\right\},(g \circ \phi)^{1 / t}\right)\right)=\infty .
$$

We will show that this implies the conclusion.

Let $V^{\perp}$ denote an orthogonal complement of $V$ in $\mathrm{F}\left(\left(X^{-1}\right)\right)^{h}$. For any $x_{0} \in V^{\perp}$ and any function $\phi: \mathbf{R}_{+} \rightarrow \mathbf{R}_{+}$, we define a subset $\Lambda_{x_{0}}(\phi)$ of the affine space $V+x_{0}$ by letting

$$
\begin{aligned}
\Lambda_{x_{0}}(\phi)=\left\{x \in V+x_{0}: d_{V+x_{0}}\left(R_{\alpha}\right.\right. & \left.\cap\left(V+x_{0}\right), x\right) \\
& \left.<\phi\left(\beta_{\alpha}\right) \text { for infinitely many } R_{\alpha}\right\},
\end{aligned}
$$

where $d_{V+x_{0}}$ denotes the induced metric on the affine space $V+x_{0}$.

As (9) holds for any ball, we get from Fubini's Theorem that there is a set $S \subseteq V^{\perp}$ of full $l$-dimensional Haar measure such that for each $x_{0} \in S$,

$$
\mathscr{H}^{t}\left(B \cap\left(V+x_{0}\right) \cap \Lambda_{x_{0}}(g \circ \phi)^{1 / t}\right)=\mathscr{H}^{t}\left(B \cap\left(V+x_{0}\right)\right) .
$$

Let $x_{0} \in S$ be fixed. 
A consequence of assumption (i) and (ii) is that the intersection between a set $R_{\alpha}$ and $V+x_{0}$ must consist of a single point, $c_{\alpha}$ say. Furthermore, it is a consequence of (ii) that for $C=\sup _{\alpha \in J} \operatorname{diam}\left(V \cap \Delta\left(R_{\alpha}, 1\right)\right)$, for any $\alpha$ and for any $r>0$

$$
\begin{array}{r}
B\left(c_{\alpha}, r\right) \cap\left(V+x_{0}\right) \subseteq\left\{x \in \mathrm{F}\left(\left(X^{-1}\right)\right)^{h}: d\left(R_{\alpha}, x\right)<r\right\} \cap\left(V+x_{0}\right) \\
\subseteq B\left(c_{\alpha}, C r\right) \cap\left(V+x_{0}\right)
\end{array}
$$

Hence, the set on the left hand side of (11) is contained within the lim sup set of a sequence of balls in $V+x_{0}$, which in turn must have full measure,

$$
\mathscr{C}^{t}\left(B \cap\left(V+x_{0}\right) \cap \lim \sup B\left(c_{\alpha}, C\left(g\left(\phi\left(\beta_{\alpha}\right)\right)\right)^{1 / t}\right)=\mathscr{H}^{t}\left(B \cap\left(V+x_{0}\right)\right) .\right.
$$

Now, Lemma 2.1 implies that we may take $C=1$ in (13), so that

$$
\mathscr{H}^{t}\left(B_{x_{0}} \cap \lim \sup B\left(c_{\alpha},\left(g\left(\phi\left(\beta_{\alpha}\right)\right)\right)^{1 / t}\right)=\mathscr{H}^{t}\left(B_{x_{0}}\right),\right.
$$

where $B_{x_{0}}=B \cap\left(V+x_{0}\right)$ is a ball in $V+x_{0}$.

Now, we apply Theorem 2.5 to the metric space $V+x_{0}$ to conclude from (14) that

$$
\mathscr{H}^{g}\left(B_{x_{0}} \cap \lim \sup B\left(c_{\alpha}, \phi\left(\beta_{\alpha}\right)\right)=\mathscr{H}^{g}\left(B_{x_{0}}\right)=\infty .\right.
$$

Using again (12), we get that for each $x_{0} \in S$,

$$
\mathscr{H}^{g}\left(B_{X_{0}} \cap \Lambda_{x_{0}}(\phi)\right)=\infty .
$$

Lemma 2.8 now implies the theorem.

\subsection{A result from probability}

In deriving asymptotic formulae, we need the following result, which originates in Rademacher's work. The version quoted here is from Sprindžuk's monograph [22]. It is phrased in a more general language than the rest of the present paper. Specialisation to the present setup will be given in $\S 3$.

Lemma 2.9. Let $(\Omega, \mathscr{B}, \mu)$ be a probability space, let $\left(X_{k}\right)$ be a sequence of non-negative measurable functions, and let $\left(f_{k}\right)$ and $\left(\tau_{k}\right)$ be sequences of real numbers with

$$
0 \leq f_{k} \leq \tau_{k} \leq 1, \quad k=1,2, \ldots
$$

Suppose that there is a constant $C>0$ such that for any pair of integers $m, n$ with $m<n$,

$$
\int_{\Omega}\left(\sum_{m<k \leq n} X_{k}(\omega)-\sum_{m<k \leq n} f_{k}\right)^{2} d \mu(\omega) \leq C \sum_{m<k \leq n} \tau_{k} .
$$


Then for any $\epsilon>0$, for almost all $\omega \in \Omega$,

$$
\sum_{k=1}^{n} X_{k}(\omega)=\sum_{k=1}^{n} f_{k}+O\left(T^{1 / 2}(n) \log ^{3 / 2+\epsilon} T(n)\right),
$$

where $T(n)=\sum_{k=1}^{n} \tau_{k}$.

\section{Asymptotic formulae}

Having assembled the necessary tools, we now embark on the proofs of the Diophantine results.

Proof of Theorem 1.1. We deduce the result from Lemmas 2.2, 2.3, 2.4 and 2.9. With reference to Lemma 2.9, let $(\Omega, \mathscr{B}, \mu)$ be $\left(\operatorname{Mat}_{m, n}(\mathscr{I}), \mathscr{B}, \mu\right)$, where $\mathscr{B}$ is the Borel $\sigma$-algebra and $\mu$ is the $m n$-fold product of the normalised Haar measure. We will use $\mathbf{q} \in \mathrm{F}[X]^{m}$ as the indexing parameter rather than $k$.

Suppose first that $m \geq 2$. For each $\mathbf{q} \in \mathrm{F}[X]^{m}$, let $X_{\mathbf{q}}: \operatorname{Mat}_{m, n}(\mathscr{I}) \rightarrow \mathbf{R}_{\geq 0}$ be the characteristic function on the set $B(\mathbf{q}, \psi(|\mathbf{q}|))$, let $f_{\mathbf{q}}=\psi(|\mathbf{q}|)^{n}$ and let $\tau_{\mathbf{q}}=\psi(|\mathbf{q}|)^{n} d(\mathbf{q})$, where $d(\mathbf{q})$ denotes the number of common factors in $\mathrm{F}[X]$ of the coordinates of $\mathbf{q}$.

We need to check the assumptions of Lemma 2.9, so consider the integral

$$
\int_{\Omega}\left(\sum_{q^{s}<|\mathbf{q}| \leq q^{t}} X_{\mathbf{q}}(A)-\sum_{q^{s}<k \leq q^{t}} f_{\mathbf{q}}\right)^{2} d \mu(A),
$$

where $s<t$ are integers. We expand the square to obtain a double sum over $\mathbf{q}$ and $\mathbf{q}^{\prime}$ over the same range of norms. We then split this sum up into the cases when $\mathbf{q}$ and $\mathbf{q}^{\prime}$ are linearly independent and linearly dependent respectively.

Consider first the case when $\mathbf{q} \nVdash \mathbf{q}^{\prime}$, i.e., the case of linear independence. In this case, the contribution to the integral (17) is

$$
\begin{aligned}
\int_{\Omega} \sum_{\mathbf{q} \nVdash \mathbf{q}^{\prime}}\left(X_{\mathbf{q}}(A)-f_{\mathbf{q}}\right)\left(X_{\mathbf{q}^{\prime}}(A)-f_{\mathbf{q}^{\prime}}\right) d \mu(A) \\
=\sum_{\mathbf{q} \nVdash \mathbf{q}^{\prime}}\left(\int_{\Omega} X_{\mathbf{q}}(A) X_{\mathbf{q}^{\prime}}(A) d \mu(A)-f_{\mathbf{q}^{\prime}} \int_{\Omega} X_{\mathbf{q}}(A) d \mu(A)\right. \\
\left.\quad-f_{\mathbf{q}} \int_{\Omega} X_{\mathbf{q}^{\prime}}(A) d \mu(A)+f_{\mathbf{q}} f_{\mathbf{q}^{\prime}}\right) \\
=\sum_{\mathbf{q} \nVdash \mathbf{q}^{\prime}}\left(f_{\mathbf{q}} f_{\mathbf{q}^{\prime}}-f_{\mathbf{q}^{\prime}} f_{\mathbf{q}^{\prime}}-f_{\mathbf{q}^{\prime}} f_{\mathbf{q}^{\prime}}+f_{\mathbf{q}} f_{\mathbf{q}^{\prime}}\right)=0,
\end{aligned}
$$


where we have used Lemma 2.2 and Lemma 2.3 to evaluate the integrals. Hence, we only get contributions to (17) from the linearly dependent case.

Consider now the linearly dependent case. It is evident that in order for $\mathbf{q}$ and $\mathbf{q}^{\prime}$ with $|\mathbf{q}| \geq|\mathbf{q}|^{\prime}$ to be linearly dependent, there must be a $d \in \mathrm{F}[X]$ such that $d \mathbf{q}^{\prime}=\mathbf{q}$. In other words, there must be a $d$, which divides all coordinates of $\mathbf{q}$, and there is a linearly dependent vector $\mathbf{q}^{\prime}$ for each such $d$. Also, we find in this case that

$$
\mu(B(\mathbf{q}, \psi(|\mathbf{q}|)) \cap B(d \mathbf{q}, \psi(|d \mathbf{q}|))) \leq \mu\left(B(\mathbf{q}, \psi(|\mathbf{q}|))=\psi(|\mathbf{q}|)^{n},\right.
$$

since $\psi$ is non-increasing.

Using (18) and Lemma 2.2,

$$
\begin{aligned}
& \int_{\Omega} \sum_{\mathbf{q} \| \mathbf{q}^{\prime}}\left(X_{\mathbf{q}}(A)-f_{\mathbf{q}}\right)\left(X_{\mathbf{q}^{\prime}}(A)-f_{\mathbf{q}^{\prime}}\right) d \mu(A) \\
& \leq \sum_{\mathbf{q}} \sum_{d \mid q_{i}, 1 \leq i \leq m}\left(\int_{\Omega} X_{\mathbf{q}}(A) X_{d \mathbf{q}}(A) d \mu(A)-f_{d \mathbf{q}} \int_{\Omega} X_{\mathbf{q}}(A) d \mu(A)\right. \\
& \left.\quad-\quad f_{\mathbf{q}} \int_{\Omega} X_{d \mathbf{q}}(A) d \mu(A)+f_{\mathbf{q}} f_{d \mathbf{q}}\right) \\
& \leq \sum_{\mathbf{q}} \sum_{d \mid q_{i}, 1 \leq i \leq m} \mu(B(\mathbf{q}, \psi(|\mathbf{q}|)) \cap B(d \mathbf{q}, \psi(|d \mathbf{q}|))) \\
& \leq \sum_{\mathbf{q}} d(\mathbf{q}) \psi(|\mathbf{q}|)^{n}=\sum_{\mathbf{q}} \tau_{\mathbf{q}} .
\end{aligned}
$$

By Lemma 2.9, this gives an asymptotic formula with the correct main term.

It remains to show that the error term is of the same order of magnitude as that in the statement of the theorem. In other words, it suffices to show that

$$
T(Q)=\sum_{|\mathbf{q}| \leq Q} d(\mathbf{q}) \psi(|\mathbf{q}|)^{n} \ll \sum_{\mathbf{q} \leq Q} \psi(\mathbf{q})^{n}=\Phi(Q) .
$$

This was done in [7] in the final step of the proof of the main theorem of that paper. This completes the proof.

We now prove the doubly metric statement of Corollary 1.2.

Proof OF Corollary 1.2. We deduce Corollary 1.2 in the case $m \geq 2$ by applying Fubini's Theorem to the indicator of the exceptional set and using Theorem 1.1. Hence, it remains only to deduce the result for the case $m=1$. Here, the result follows from Lemma 2.4 and Lemma 2.9 on letting $X_{\mathbf{q}}(A, \mathbf{y})$ 
be the characteristic function on $\hat{B}(\mathbf{q}, \psi(|\mathbf{q}|))$ and $f_{\mathbf{q}}=\tau_{\mathbf{q}}=\psi(|\mathbf{q}|)$. The deduction is similar to that in the proof of Theorem 1.1, but slightly simpler.

REMARK. The asymptotic formulae can be generalised to give asymptotics along subsequences, i.e., under restriction to the case when $\mathbf{q} \in \mathscr{S} \in \mathrm{F}[X]^{m}$. This extends results in [10]. In both cases, the main term is a volume sum as above, and the error term will be bounded by the square root of the main term times a power of the logarithm of the main term. In the case when $m=1$, this is immediate. If $m \geq 2$, the error term requires a little work. We leave the details to the interested reader.

\section{Zero-one and zero-infinity laws}

Proving Theorem 1.3 and Corollary 1.4 from Theorem 1.1 and Corollary 1.2 is an easy exercise. Indeed, the series which determines whether the measure is null or full is the same as the main term of the asymptotic formulae. Furthermore, if the series in question converges, the error term is also bounded. Hence, the asymptotic formulae immediately imply Theorem 1.3 and 1.4.

It remains for us to prove the Hausdorff measure results. We derive the singly metric statement of Theorem 1.5 and give a sketch of the doubly metric case of Corollary 1.6. The proof in the latter case is very similar, and the details are left to the interested reader.

There are two parts of the theorem. The case when the series converges uses a covering argument.

Proof of Theorem 1.5 (CONVERgence Part). For each $\mathbf{q}$, we consider the affine planes $\mathbf{q} A=\mathbf{p}, \mathbf{p} \in \mathrm{F}[X]^{n}$. Exactly $|\mathbf{q}|^{n}$ of these intersect non-trivially with $\mathscr{I}^{n m}$. Inside each, we choose points at distance an integer multiple of $\psi(|\mathbf{q}|) /|\mathbf{q}|$ of each other. Evidently, for each $\mathbf{p}$, we may cover the set

$$
\left\{A \in \operatorname{Mat}_{n, m}(\mathscr{I}): \mathbf{q} A=\mathbf{p}\right\}
$$

with $(\psi(|\mathbf{q}|) /|\mathbf{q}|)^{(m-1) n}$ balls centred at these points with radius $\psi(|\mathbf{q}|) /|\mathbf{q}|$.

Now, for each $N \geq 1$, the set $W_{m, n}(\psi, \mathbf{y})$ is contained in the union of these balls, where $|\mathbf{q}| \geq N$ and $\mathbf{p}$ runs over the allowed range. Using this as a natural cover in the definition of Hausdorff measure yields,

$$
\begin{aligned}
\mathscr{C}^{f}\left(W_{m, n}(\psi, \mathbf{y})\right) & \leq \sum_{\substack{\mathbf{q} \in[X] X^{m} \\
|\mathbf{q}| \geq N}}|\mathbf{q}|^{n}\left(\frac{\psi(|\mathbf{q}|)}{|\mathbf{q}|}\right)^{(m-1) n} f\left(\frac{\psi(|\mathbf{q}|)}{|\mathbf{q}|}\right) \\
& =\sum_{\substack{\mathbf{q} \in[X]]^{m} \\
|\mathbf{q}| \geq N}} g\left(\frac{\psi(|\mathbf{q}|)}{|\mathbf{q}|}\right)|\mathbf{q}|^{n} .
\end{aligned}
$$


As this holds true for every $N$, and as the last series above is assumed to converge, we have the conclusion.

Note that if we apply the covering argument to the dimension function $f(r)=r^{m n}$, we obtain a direct proof of the convergence case of Theorem 1.3, independent of the asymptotic formula.

In order to obtain the divergence part of Theorem 1.5, we will use the slicing technique from Section 2.4. Essentially, we follow Beresnevich and Velani [3] in the deduction.

Proof of Theorem 1.5 (Divergence CaSe). We will deduce the result from Theorem 1.3 and Theorem 2.6. Our first task is to get Theorem 1.3 into a form, where the Slicing Theorem applies. We will make some very crude estimates in the process, which no doubt could be improved upon, although this would have no impact on the result. Without loss of generality, we can assume that $r^{-m n} f(r) \rightarrow \infty$ as $r \rightarrow 0$, since otherwise the result is a trivial consequence of Theorem 1.3. See also (8) above for details on this assumption.

First, let $\phi(r)=\psi(r) / r^{m}$. We also restrict the family of resonant sets to those defined by $\mathbf{q}=\left(q_{1}, \ldots, q_{m}\right)$ with $|\mathbf{q}|=\left|q_{1}\right|$. With reference to the framework of Section 2.4, $h=m n$ and $\left\{R_{\alpha}\right\}$ is the family of $(m-1) n$ dimensional affine planes defined by the conditions

$$
\mathbf{q} A-\mathbf{y}-\mathbf{p}=0 \quad \text { and } \quad|\mathbf{q}|=\left|q_{1}\right| .
$$

It is straightforward to prove that

$$
W\left(\left\{R_{\alpha}\right\}, \phi\right) \subseteq W_{m, n}(\psi, \mathbf{y}) .
$$

Hence, to show that the Hausdorff $f$-measure of the right hand side is infinite, it suffices to show that the left hand side has infinite Hausdorff $f$-measure.

Repeating the arguments used in the proof of Theorem 1.1, we easily show that if $\sum_{\mathbf{q} \in \mathrm{F}[X]^{m}} \phi(|\mathbf{q}|)^{n}|\mathbf{q}|^{n}=\infty$, then the set $W\left(\left\{R_{\alpha}\right\}, \phi\right)$ is full with respect to Haar measure. Note that this divergence condition is equivalent to that of Theorem 1.3.

Again with reference to the framework of Section 2.4, $l=(m-1) n$ and $t=n$. In order to apply the Slicing Theorem, we need an $n$-dimensional linear subspace, which intersects all the $R_{\alpha}$ non-trivially, and such that condition (ii) of Theorem 2.6 holds. Evidently, the subspace

$$
V=\left\{A=\left(a_{i j}\right) \in \operatorname{Mat}_{m, n}\left(\mathrm{~F}\left(\left(X^{-1}\right)\right)\right): a_{i j}=0 \text { for } j \geq 2\right\},
$$

i.e., the set of $m \times n$ matrices with zeros off the first row, satisfies the required assumptions. 
Recall that we have assumed that

$$
\sum_{\mathbf{q} \in \mathrm{F}[X]^{m} \backslash\{0\}} g\left(\frac{\psi(|\mathbf{q}|)}{|\mathbf{q}|}\right)|\mathbf{q}|^{n}=\sum_{\mathbf{q} \in \mathrm{F}[X]^{m} \backslash\{0\}}\left(g(\phi(|\mathbf{q}|))^{1 / n}\right)^{n}|\mathbf{q}|=\infty .
$$

Hence, $W\left(\left\{R_{\alpha}\right\}, \phi\right)$ is full, so that for any ball $B \subseteq \operatorname{Mat}_{m, n}\left(\mathrm{~F}\left(\left(X^{-1}\right)\right)\right)$,

$$
\mathscr{H}^{m n}\left(B \cap W\left(\left\{R_{\alpha}\right\},(g \circ \phi)^{1 / n}\right)\right)=\mathscr{H}^{m n}(B) .
$$

It then follows from Theorem 2.6 that

$$
\mathscr{H}^{f}\left(B \cap W\left(\left\{R_{\alpha}\right\}, \phi\right)\right)=\mathscr{H}^{f}(B)=\infty .
$$

The latter equality follows as $r^{-m n} f(r) \rightarrow \infty$ as $r \rightarrow 0$.

We now sketch a proof of the doubly metric result.

Sketch of THE PROOF OF Corollary 1.6. The proof is essentially the same as the proof of Theorem 1.5, except that we let $\mathbf{y}$ vary in the definition of the $R_{\alpha}$. For the convergence case, the argument carries over, with the only modification that we now need $(\psi(|\mathbf{q}|)|\mathbf{q}|)^{m n}$ balls to cover each resonant neighbourhood.

For the divergence case, we also follow the proof of Theorem 1.5, but we obtain new parameters, so that with reference to the framework of Section 2.4, $h=(m+1) n, l=m n$ and $k=n$. The subspace $V$ is still an appropriate subspace for the Slicing Theorem to work. The divergence condition which we used from Theorem 1.3 is replaced with that of Corollary 1.4. Otherwise, the proof is identical.

Finally, we deduce the results on Hausdorff dimension.

Proof of Corollary 1.7. Note that by assumption, for any $\epsilon>0$, there is an $r_{0}>0$ such that if $q^{k} \geq r_{0}$, then

$$
|\mathbf{q}|^{-\lambda-\epsilon} \leq \psi(|\mathbf{q}|) \leq|\mathbf{q}|^{-\lambda+\epsilon} .
$$

Hence,

$$
W_{m, n}\left(r \mapsto r^{-\lambda-\epsilon}, \mathbf{y}\right) \subseteq W_{m, n}(\psi, \mathbf{y}) \subseteq W_{m, n}\left(r \mapsto r^{-\lambda+\epsilon}, \mathbf{y}\right),
$$

and it suffices to find the Hausdorff dimension of the leftmost and rightmost sets above.

However, using the definition of Hausdorff dimension together with Theorem 1.5 , we find that for any $v>m / n$,

$$
\operatorname{dim}_{H}\left(W_{m, n}\left(r \mapsto r^{-v}, \mathbf{y}\right)\right)=(m-1) n+\frac{m+n}{1+v} .
$$


Hence,

$$
(m-1) n+\frac{m+n}{1+v+\epsilon} \leq \operatorname{dim}_{H}\left(W_{m, n}(\psi, \mathbf{y})\right) \leq(m-1) n+\frac{m+n}{1+v-\epsilon} .
$$

As $\epsilon>0$ is arbitrary, the conclusion follows for $\lambda>m / n$.

To obtain the result for $\lambda \leq m / n$, note that we can get a lower bound as close to $m n$ as we wish. Since $m n$ is an upper bound on the Hausdorff dimension of any set contained in $\operatorname{Mat}_{m, n}(\mathscr{I})$, we get the result in this case.

Proof Of Corollary 1.8. This proof goes exactly as the proof of Corollary 1.7 with Corollary 1.6 in place of Theorem 1.5.

\section{Concluding remarks}

Although we have covered most possible cases to consider in the theory, there are still a few open cases left. To conclude the paper, we summarise some of these open problems.

The case when $m=1$ has not been completely resolved, in the sense that the singly metric asymptotic formula Theorem 1.1 has not been shown to be valid for $m=1$. It is natural to conjecture that it does indeed hold. In order to prove this, one would need to prove a lemma in the spirit of Lemma 2.4, where the doubly metric resonant neighbourhoods are replaced with the singly metric ones.

Of course, strict equality for all different values of $\mathbf{q}$ and $\mathbf{q}^{\prime}$ is not needed. In fact, it would be possible to prove the result if we could prove an approximate equality for $\mathbf{q}$ running in a sufficiently dense set in $\mathrm{F}[X]$. We have not attempted to obtain such a result in this paper and have only showed the doubly metric result for $m=1$. However, especially in view of the results of Berthé, Nakada and Natsui [4], there is no reason to suspect that Theorem 1.1 breaks down for $m=1$, although proving it would add considerably to the technicality of the paper.

Another problem for $m=1$, which is possibly more tractable, is the zeroone law of Theorem 1.3 and the Hausdorff measure zero-infinity law of Theorem 1.5. Again, the assumption that $m \geq 2$ is somewhat artificial. In this case, it comes from the fact that we deduced these theorems from the asymptotic formula, Theorem 1.1. However, looking at the proof of the convergence case of Theorem 1.7, we find that the assumption is not needed. This suggests that an alternative approach is perhaps possible, and this is indeed the case.

In order to bypass the technical difficulties encountered when extending the asymptotic formula to this case, one is tempted to use the notion of ubiquitous systems, see e.g. [1]. This can indeed be accomplished using only the tools from this paper in the case $m \geq 2$. In fact, we could in this case replace the 
equality in Lemma 2.3 with a $\leq$, and would only need this lemma to hold for a suitably dense subset and up to a universal multiplicative constant on the right hand side. Obtaining such a result for the case $m=1$ would be considerably less technical than getting the asymptotic equality needed for the asymptotic formula. Nevertheless, we have chosen not to pursue this any further in this paper for the sake of the presentation.

A variant of inhomogeneous Diophantine approximation in the singly metric setup puts the emphasis on the inhomogeneous term by fixing $A$ and considering instead the set of $\mathbf{y}$ for which certain inequalities have infinitely many solutions. This study which goes back to Kurzweil [11] has recently had a revival in the case of real numbers. Whether analogues of these results hold for formal power series appears to be open, and investigating this remains a challenge.

ACKNOWLEDGEMENTS. I thank Maurice Dodson for encouraging me to write the present paper and the referee for his helpful comments.

\section{REFERENCES}

1. Beresnevich, V., Dickinson, D., and Velani, S., Measure theoretic laws for lim sup sets, Mem. Amer. Math. Soc. 179 (2006), no. 846.

2. Beresnevich, V., and Velani, S., A mass transference principle and the Duffin-Schaeffer conjecture for Hausdorff measures, Ann. of Math. (2) 164 (2006), 971-992.

3. Beresnevich, V., and Velani, S., Schmidt's theorem, Hausdorff measures, and slicing, Int. Math. Res. Not. 2006, Art. ID 48794.

4. Berthé, V., Nakada, H., and Natsui, R., Asymptotic behavior of the number of solutions for non-Archimedean Diophantine approximations with restricted denominators, Finite Fields Appl. 14 (2008), 849-866.

5. Dickinson, H., A note on the theorem of Jarník-Besicovitch, Glasgow Math. J. 39 (1997), 233-236.

6. Dodson, M. M., A note on metric inhomogeneous Diophantine approximation, J. Austral. Math. Soc. (A) 62 (1997), 175-185.

7. Dodson, M. M., Kristensen, S., and Levesley, J., A quantitative Khintchine-Groshev type theorem over a field of formal series, Indag. Math. (N.S.) 16 (2005), 171-177.

8. Khintchine, A., Einige Sätze über Kettenbrüche, mit Anwendungen auf die Theorie der Diophantischen Approximationen, Math. Ann. 92(1924), 115-125.

9. Kristensen, S., On well-approximable matrices over a field of formal series, Math. Proc. Cambridge Philos. Soc. 135 (2003), 255-268.

10. Kristensen, S., A metric theorem for restricted Diophantine approximation in positive characteristic, Acta Arith. 124 (2006), 159-175.

11. Kurzweil, J., On the metric theory of inhomogeneous Diophantine approximations, Studia Math. 15 (1955), 84-112.

12. Levesley, J., A general inhomogeneous Jarnik-Besicovitch theorem, J. Number Theory 71 (1998), 65-80.

13. Ma, C., and Su, W.-Y., Inhomogeneous Diophantine approximation over the field of formal Laurent series, Finite Fields Appl. 14 (2008), 361-378. 
14. Mahler, K., An analogue to Minkowski's geometry of numbers in a field of series, Ann. of Math. (2) 42 (1941), 488-522.

15. de Mathan, B., Approximations diophantiennes dans un corps local, Bull. Soc. Math. France Suppl. Mém. 21 (1970).

16. Mattila, P., Geometry of Sets and Measures in Euclidean Spaces. Fractals and Rectifiability, Cambridge Studies in Advanced Math. 44, Cambridge Univ. Press, Cambridge 1995.

17. Nachbin, L., The Haar Integral, Van Nostrand, Princeton, N.J. 1965.

18. Niederreiter, H., and Vielhaber, M., Linear complexity profiles: Hausdorff dimensions for almost perfect profiles and measures for general profiles, J. Complexity 13 (1997), 353383.

19. Schmidt, W. M., A metrical theorem in Diophantine approximation, Canad. J. Math. 12 (1960), 619-631.

20. Schmidt, W. M., Metrical theorems on fractional parts of sequences, Trans. Amer. Math. Soc. 110 (1964), 493-518.

21. Sprindžuk, V. G., Mahler's Problem in Metric Number Theory, Translations of Math. Monographs 25, Amer. Math. Soc., Providence, R.I. 1969.

22. Sprindžuk, V. G., Metric Theory of Diophantine Approximations. Winston, Washington, D.C. 1979.

DEPARTMENT OF MATHEMATICAL SCIENCES

FACULTY OF SCIENCE

UNIVERSITY OF AARHUS

NY MUNKEGADE 118

DK-8000 AARHUS C

DENMARK

E-mail: sik@imf.au.dk 